\title{
TOTALLY SMOOTH RENORMINGS
}

\author{
EVE OJA, TAURI VIIL, AND DIRK WERNER
}

\begin{abstract}
We study the problem of totally smooth renormings of Banach spaces and provide such renormings for spaces which are weakly compactly generated. We also consider renormings for $(a, B, c)$-ideals.
\end{abstract}

\section{INTRODUCTION}

Let $X$ be a Banach space. Following Phelps $[\mathrm{P}$, we say that a subspace $X$ of a Banach space $Z$ has property $U$ in $Z$ if every functional $x^{*} \in X^{*}$ has a unique norm-preserving extension $z^{*} \in Z^{*}$. Following Liao and Wong [LW], we say that $X$ is totally smooth in $Z$ if every closed subspace $Y$ of $X$ has property $U$ in $Z$. If $Z=X^{* *}$, then we say that $X$ has property $U$ in its bidual or, respectively, that $X$ is totally smooth in its bidual. Banach spaces with property $U$ in their biduals are also known as Hahn-Banach smooth spaces [S].

The notion of total smoothness in the bidual was essentially considered already in 1977 by Sullivan [S]. It was further studied in [OPV], where several geometrical conditions equivalent to total smoothness were proved.

Let $\pi_{X}: X^{* * *} \rightarrow X^{* * *}$ denote the natural projection onto the dual space $X^{*}$. It is known (see [02] or, e.g., [04, p. 21]) that $X$ has property $U$ in its bidual $X^{* *}$ if and only if $X$ has the strong uniqueness property $S U$ in $X^{* *}$, meaning that the following condition holds: for $x^{* * *} \in X^{* * *}$,

$$
\left\|\pi_{X} x^{* * *}\right\|=\left\|x^{* * *}\right\| \quad \Rightarrow \quad \pi_{X} x^{* * *}=x^{* * *} .
$$

A Banach space $X$ is called an $M$-ideal in its bidual if the equality

$$
\left\|x^{* * *}\right\|=\left\|\pi_{X} x^{* * *}\right\|+\left\|x^{* * *}-\pi_{X} x^{* * *}\right\|
$$

holds for every $x^{* * *} \in X^{* * *}$. The notion of $M$-ideals was introduced by Alfsen and Effros in $\mathrm{AE}$ and has since then been studied by many authors (see, e.g., the monograph [HWW] for results and references).

The motivation for this paper comes from the following observation in OPV] Remark 2.8], which is based on [HWW, Theorem III.4.6].

Observation. If $X$ is an $M$-ideal in its bidual $X^{* *}$, then $X$ admits an equivalent norm under which $X$ is totally smooth and is still an $M$-ideal in its bidual.

As, clearly, being an $M$-ideal implies property $U$, the natural question arises whether the $M$-ideal condition in the Observation could be relaxed.

Problem 1.1. If $X$ has property $U$ in its bidual $X^{* *}$, then does $X$ admit an equivalent norm under which $X$ is totally smooth in its bidual?

Date: 17.7 .2018 .

2010 Mathematics Subject Classification. 46B03, 46B04, 46B20.

Key words and phrases. Totally smooth Banach space, property $U,(a, B, c)$-ideals, renormings. 
Let us note at once (see Example 3.5 below) that there exist Banach spaces that admit an equivalent norm under which they are totally smooth, but they do not admit any equivalent norm under which they are $M$-ideals in their biduals.

Now, we recall that according to the Taylor-Foguel theorem (see [T] and [Fo]), every subspace $Y$ of $X$ has property $U$ in $X$ if and only if the dual space $X^{*}$ is strictly convex, i.e., its unit sphere $S_{X^{*}}$ contains no non-trivial line segments.

Thus, relying on the Taylor-Foguel theorem, we can characterize totally smooth spaces by the strict convexity of the dual space as follows.

Theorem 1.2 (see $[\mathrm{LW}]$ ). A Banach space $X$ is totally smooth in its bidual $X^{* *}$ if and only if $X$ has property $U$ in $X^{* *}$ and the dual space $X^{*}$ is strictly convex.

Therefore, Problem 1.1 is equivalent to the following problem.

Problem 1.3. If $X$ has property $U$ in its bidual $X^{* *}$, then does $X$ admit an equivalent norm under which the dual space $X^{*}$ is strictly convex and $X$ still has property $U$ in its bidual?

Recall that if $X$ is separable and has property $U$ in $X^{* *}$, then $X^{*}$ is separable (see Theorem 2.1 below). It was observed by Sullivan in [S, p. 321] that an application of the Kadets-Klee renorming theorem solves Problem 1.3 (and thus also Problem 1.1) fully and positively for separable spaces. This theorem provides a Banach space having a separable dual with an equivalent norm whose dual norm is strictly convex and has the property that the weak* topology and the norm topology coincide on the (new) dual sphere. A proof of this renorming theorem can be found in Di, pp. 113-117]; it first appeared in Klee's paper [K12] relying on work by Kadets in Ka.

In Section 3, using a simple Klee-type renorming [Kl1, we give a partial positive answer to Problem 1.3, and thus to Problem 1.1, in the general case (see Theorem 3.2. In particular, our results also provide an alternative proof for the separable case. In Section 4, we come back to the Observation and we show that, under natural assumptions, its claim holds for $(a, B, c)$-ideals, which are a far-reaching generalization of $M$-ideals, encompassing in particular $u$ - and $h$-ideals.

Our notation is standard. We consider Banach spaces over the scalar field $\mathbb{K}=\mathbb{R}$ or $\mathbb{K}=\mathbb{C}$. For a Banach space $X, B_{X}$ is the closed unit ball and $S_{X}$ is the unit sphere of $X$. By $\overline{\operatorname{span}}\left(x_{i}\right)$, we denote the closed linear span of the elements $x_{i}$. For a subspace $X$ of $Z, X^{\perp}=\left\{z^{*} \in Z^{*}: z^{*}{ }_{\mid X}=0\right\}$ is the annihilator. The density character of the space $X$ is denoted by dens $X$. For a bounded linear operator $T$, $T^{*}$ is the adjoint operator, $\operatorname{ran} T$ is the range, and $\operatorname{ker} T$ is the kernel of $T$.

\section{UsEFul RESULts}

In this section, we note some useful results regarding property $U$ and very smooth norms.

Recall that a Banach space $X$ is an Asplund space if every separable subspace $Y$ of $X$ has a separable dual space $Y^{*}$. The following result (implicitly in [SS, Theorem 15]) is well known.

Theorem 2.1. A Banach space $X$ with property $U$ in its bidual $X^{* *}$ is an Asplund space.

In addition, we will need the following known result for Asplund spaces. 
Theorem 2.2 (see [Fa, p. 112 and Theorem 8.3.3]). For a Banach space X, the following conditions are equivalent.

(a) $X$ has a shrinking Markushevich basis, i.e., there are $\left(x_{i}\right)_{i \in I}$ in $X$ and $\left(f_{i}\right)_{i \in I}$ in $X^{*}$ such that $I$ has the cardinality dens $X$, and

- $f_{i}\left(x_{j}\right)=\delta_{i j}$

- $\overline{\operatorname{span}}\left(x_{i}\right)=X$,

- $\overline{\operatorname{span}}\left(f_{i}\right)=X^{*}$.

(b) $X$ is weakly compactly generated (WCG) and Asplund.

Recall that a Banach space $X$ is weakly compactly generated (WCG) if $X$ is the closed linear span of some weakly compact subset of $X$. The most important result on WCG spaces is the following Amir-Lindenstrauss theorem.

Theorem 2.3 (see [AL] or, e.g., [Fa, Theorem 1.2.5]). A Banach space $X$ is weakly compactly generated if and only if there exist a set $\Gamma \neq \emptyset$ and an injective weak* to-weak continuous linear operator from $X^{*}$ to $c_{0}(\Gamma)$.

We will also make use of the notion of very smooth spaces. First, recall that a Banach space $X$ is smooth whenever for every $x \in S_{X}$, there exists a unique functional $f_{x} \in S_{X^{*}}$ such that $f_{x}(x)=1$. If $X$ is smooth, then the support mapping $x \mapsto f_{x}$ from $S_{X}$ to $S_{X^{*}}$ is norm-to-weak* continuous (see, e.g., [Di, p. 22]).

Definition 2.4 (see [DF] or, e.g., [Di, p. 31]). A smooth Banach space $X$ is called very smooth if the support mapping $x \mapsto f_{x}$ from $S_{X}$ to $S_{X^{*}}$ is norm-to-weak continuous.

It is well known that a Banach space $X$ is smooth whenever its dual space $X^{*}$ is strictly convex, but it need not be very smooth in general [S].

To prove that a renorming is very smooth, we will use the following result.

Lemma 2.5 (see [G] or, e.g., [HWW, Lemma III.2.14]). A Banach space $X$ has property $U$ in its bidual $X^{* *}$ if and only if the relative weak and weak ${ }^{*}$ topologies on $B_{X^{*}}$ coincide on $S_{X^{*}}$.

\section{Renorming of Banach spaces With Property $U$}

In order to try to solve Problem [1.3. we follow the strategy of the proof of the Observation. This proof has three steps.

(i) The Banach space $X$ has a shrinking Markushevich basis (as proved by Fabian and Godefroy [FG]).

(ii) Using the shrinking Markushevich basis, one obtains an injective weak*-toweak continuous linear operator from $X^{*}$ to $c_{0}(\Gamma)$. This allows one to equip $X$ with a rather standard equivalent norm $|\cdot|$ such that for $\widetilde{X}:=(X,|\cdot|)$, the dual norm of $\widetilde{X}^{*}$ is strictly convex (as proved already by Amir and Lindenstrauss $\mathrm{AL}$ ).

(iii) It can be shown that the renorming in (ii) is such that $\widetilde{X}$ is still an $M$-ideal in $\widetilde{X}^{* *}$ (as proved by Harmand and Rao in [HR]).

The step (iii) can be extended from the $M$-ideal case (see [HR] or [HWW, Proposition III.2.11]) to the property $U$ case by the following theorem. 
Theorem 3.1. Let $X$ be a Banach space with property $U$ in its bidual $X^{* *}$. If $Y$ is a Banach space and $T: Y \rightarrow X$ a weakly compact operator, then

$$
\left|x^{*}\right|:=\left\|x^{*}\right\|+\left\|T^{*} x^{*}\right\|, \quad x^{*} \in X^{*},
$$

is an equivalent dual norm on $X^{*}$ for which $\widetilde{X}:=(X,|\cdot|)$ has property $U$ in $\tilde{X}^{* *}$.

Moreover, if $T^{*}$ is injective and there is a strictly convex Banach space $Z$ such that $\operatorname{ran} T^{*} \subset Z \subset Y^{*}$, then $\widetilde{X}^{*}$ is strictly convex.

Proof. Since $T^{*}$ is an adjoint operator, it is weak*-to-weak* continuous, and thus the mapping $x^{*} \mapsto\left\|T^{*} x^{*}\right\|, x^{*} \in X^{*}$, is weak ${ }^{*}$ lower semicontinuous. Therefore, $|\cdot|$ is an equivalent dual norm by a well-known result of Klee [Kl1]; see, e.g., Di, p. 106].

To calculate $|\cdot|$ on the third dual $X^{* * *}$, we use the following argument due to Harmand and Rao in $\mathrm{HR}$. By definition, the operator

$$
S:\left(X^{*},|\cdot|\right) \rightarrow X^{*} \oplus_{1} Y^{*}, \quad x^{*} \mapsto\left(x^{*}, T^{*} x^{*}\right),
$$

is isometric; hence

$$
S^{* *}:\left(X^{* * *},|\cdot|\right) \rightarrow X^{* * *} \oplus_{1} Y^{* * *}
$$

is isometric too. One can easily see that $S^{* *} x^{* * *}=\left(x^{* * *}, T^{* * *} x^{* * *}\right)$, so

$$
\left|x^{* * *}\right|=\left\|x^{* * *}\right\|+\left\|T^{* * *} x^{* * *}\right\|, \quad x^{* * *} \in X^{* * *} .
$$

By the weak compactness of $T^{*}$, we get that $\operatorname{ran} T^{* * *} \subset Y^{*}$, hence $\pi_{Y} T^{* * *}=T^{* * *}$. Since $\pi_{Y} T^{* * *}=T^{* * *} \pi_{X}$, we conclude that $T^{* * *}=T^{* * *} \pi_{X}$.

We need to show that the natural projection $\pi_{X} \in \mathcal{L}\left(X^{* * *}\right)$ satisfies the condition

$$
\left|\pi_{X} x^{* * *}\right|=\left|x^{* * *}\right| \Rightarrow \pi_{X} x^{* * *}=x^{* * *} .
$$

Let $x^{* * *} \in X^{* * *}$ be such that $\left|\pi_{X} x^{* * *}\right|=\left|x^{* * *}\right|$. Then

$$
\begin{aligned}
0 & =\left|\pi_{X} x^{* * *}\right|-\left|x^{* * *}\right| \\
& =\left\|\pi_{X} x^{* * *}\right\|+\left\|T^{* * *} \pi_{X} x^{* * *}\right\|-\left\|x^{* * *}\right\|-\left\|T^{* * *} x^{* * *}\right\| \\
& =\left\|\pi_{X} x^{* * *}\right\|-\left\|x^{* * *}\right\| .
\end{aligned}
$$

Therefore, $\left\|\pi_{X} x^{* * *}\right\|=\left\|x^{* * *}\right\|$, and thus $\pi_{X} x^{* * *}=x^{* * *}$ by property $U$ of $X$ in $X^{* *}$.

Moreover, if $T^{*}$ is injective and there is a strictly convex Banach space $Z$ such that $\operatorname{ran} T^{*} \subset Z \subset Y^{*}$, then, thanks to Klee's renorming theorem in [K11] (see, e.g., [Di, Theorem 1, p. 100]), $\widetilde{X}^{*}$ is strictly convex.

Using Theorem 3.1. we can now give a partial answer to Problem 1.1.

Theorem 3.2. If a $W C G$ Banach space $X$ has property $U$ in its bidual $X^{* *}$, then $X$ has a shrinking Markushevich basis and $X$ admits an equivalent very smooth norm under which $X$ is totally smooth in its bidual.

Proof. Since $X$ is also Asplund (see Theorem 2.1), it has a shrinking Markushevich basis (see Theorem 2.2). All we need to finish the proof is an injective weak*-to-weak continuous linear operator $S: X^{*} \rightarrow c_{0}(\Gamma)$ (for some set $\Gamma$ ). Such an operator $S$ exists according to the Amir-Lindenstrauss theorem (see Theorem 2.3). However, $S$ can be very easily constructed using our shrinking Markushevich basis. From now, we follow the proof of [HWW, Theorem III.4.6(e)]. 
Let $\left(x_{i}, f_{i}\right)_{i \in I}$ with $x_{i} \in X, f_{i} \in X^{*}$ be a shrinking Markushevich basis. Assuming $\left\|x_{i}\right\|=1$, we define an operator $S: X^{*} \rightarrow c_{0}(I)$ by

$$
x^{*} \mapsto\left(x^{*}\left(x_{i}\right)\right), \quad x^{*} \in X^{*} .
$$

It is easy to check that the operator $S$ is well-defined, injective, and weak*-to-weak continuous. In particular, $S$ is weakly compact and weak*-to-weak ${ }^{*}$ continuous when considered as an operator into $c_{0}(I)^{* *}$, for which we use the notation $\tilde{S}$. Hence, $\tilde{S}$ is the adjoint of a weakly compact operator $T: c_{0}(I)^{*} \rightarrow X$.

We now equip $c_{0}(I)$ with Day's equivalent strictly convex norm [Da] (see, e.g., [Di. p. 94]). Since $T$ satisfies the requirements in Theorem 3.1, we get an equivalent smooth norm $|\cdot|$ on $X$ whose dual norm is strictly convex and for which $X$ still has property $U$ in its bidual. The norm $|\cdot|$ on $X$ is, in fact, very smooth. Indeed, as was mentioned above, the support mapping on a smooth space is always norm-to-weak* continuous, hence for $(X,|\cdot|)$, by Lemma 2.5, the support mapping is norm-to-weak continuous, i.e., $(X,|\cdot|)$ is very smooth.

Since separable Banach spaces are WCG, Theorem 3.2 gives an alternative proof to the separable case considered by Sullivan in [S] that was mentioned in the Introduction.

Corollary 3.3. If a separable Banach space $X$ has property $U$ in its bidual $X^{* *}$, then $X$ admits an equivalent very smooth norm under which $X$ is totally smooth in its bidual.

Readers particularly interested in the separable case as expounded in the previous corollary should notice that the rather easy argument of [LT1, Proposition 1.f.3] shows that a Banach space with a separable dual admits a shrinking Markushevich basis.

In order to obtain examples of spaces having a shrinking Markushevich basis, we can use the notion of $U^{*}$-spaces, which is dual to property $U$.

Definition 3.4 (see CN1]). A Banach space $X$ is said to be a $U^{*}$-space in its bidual $X^{* *}$ if for every $x^{* * *} \in X^{* * *}$ with $\pi_{X} x^{* * *} \neq 0$,

$$
\left\|x^{* * *}-\pi_{X} x^{* * *}\right\|<\left\|x^{* * *}\right\| .
$$

In [CN1, proof of Theorem 4.4], it was observed that the proofs of [FG, Theorems 1 and 3] essentially yield that every Asplund $U^{*}$-space has a shrinking Markushevich basis and is WCG. Therefore, Theorem 3.2 applies to any $U^{*}$-space with property $U$ in its bidual. This, together with some help from the literature, will be used in the next example.

Example 3.5. Let $\Gamma$ be an infinite set and let $1<p<\infty$. The $l_{p}$-sum $l_{p}\left(c_{0}(\Gamma)\right)$ admits an equivalent very smooth norm under which $X$ is totally smooth in its bidual, but it cannot be equivalently renormed to be an $M$-ideal in its bidual.

Proof. It is well known (see, e.g., [HWW, Example III.1.4(a)]) that $c_{0}(\Gamma)$ is an $M$-ideal in its bidual. Hence, clearly, it is a $U^{*}$-space. This property extends to the

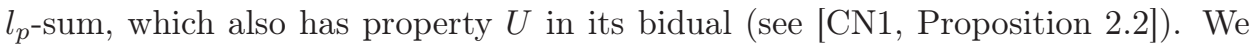
have the desired renorming of $l_{p}\left(c_{0}(\Gamma)\right)$ thanks to Theorem 3.2

Assume, for the sake of contradiction, that $X:=\left(l_{p}\left(c_{0}(\Gamma)\right),|\cdot|\right)$ is an $M$-ideal in $X^{* *}$ for some equivalent norm $|\cdot|$ on $l_{p}\left(c_{0}(\Gamma)\right)$. Since $M$-ideals in their biduals are stable by taking closed subspaces (see [HL or, e.g., [HWW, Theorem III.1.6]), 
$Y:=\left(l_{p}\left(c_{0}\right),|\cdot|\right)$ is an $M$-ideal in $Y^{* *}$. This contradicts GKS Proposition 4.4] stating that if a separable $M$-ideal $Y$ in $Y^{* *}$ has a boundedly complete Schauder decomposition $\left(Y_{n}\right)_{n=1}^{\infty}$, then all but finitely many subspaces $Y_{n}$ are reflexive. In our case, all $Y_{n}, n=1,2, \ldots$, are isomorphic to $c_{0}$ and thus non-reflexive.

\section{Renorming of $(a, B, c)$-IDEAls}

According to GKS, a closed subspace $X$ of a Banach space $Z$ is said to be an ideal in $Z$ if there is a contractive projection $P$ on $Z^{*}$ such that $\operatorname{ker} P=X^{\perp}$. In this case, the projection $P$ is called an ideal projection. If $\operatorname{ran} P$ is norming, then the ideal is called strict. If $Z=X^{* *}$ and $P=\pi_{X}$, then the ideal is called canonical. Canonical ideals are strict, but not vice versa.

Let $a, c \geq 0$ and let $B \subset \mathbb{K}$ be a compact set. If $X$ is an ideal in $Z$ with an ideal projection $P$ such that

$$
\left\|a z^{*}+b P z^{*}\right\|+c\left\|P z^{*}\right\| \leq\left\|z^{*}\right\| \quad \forall b \in B
$$

for all $z^{*}$ in $Z^{*}$, then $X$ is said to be an $(a, B, c)$-ideal in $Z$. The $(a, B, c)$-ideals were introduced in [06] (see also [05]), but got their name later in $\mathrm{OP}$. This approach unifies all previously studied special cases of ideals. For instance, it is easy to see that $M$-ideals coincide with $(1,\{-1\}, 1)$-ideals, $u$-ideals coincide with $(1,\{-2\}, 0)$-ideals, and $h$-ideals are the same as $\left(1,\left\{-(1+\lambda): \lambda \in S_{\mathbb{C}}\right\}, 0\right)$-ideals. The notions $u$ - and $h$-ideals have been deeply studied in GKS].

In the context of $(a, B, c)$-ideals, the Observation (from the Introduction) triggers the following natural question (cf. Problem 1.1).

Problem 4.1. If $X$ is an $(a, B, c)$-ideal with property $U$ in its bidual $X^{* *}$, then does $X$ admit an equivalent norm under which $X$ is totally smooth and is still an $(a, B, c)$-ideal in its bidual?

Similarly to the property $U$ case, we prove that the renorming from step (iii) from the proof of the Observation can be extended to canonical $(a, B, c)$-ideals. Concerning the special case of $M$-ideals, recall that an $M$-ideal in its bidual is always canonical.

Theorem 4.2. Let $X$ be a Banach space which is a canonical $(a, B, c)$-ideal in its bidual $X^{* *}$. If $Y$ is a Banach space and $T: Y \rightarrow X$ is a weakly compact operator, then

$$
\left|x^{*}\right|:=\left\|x^{*}\right\|+\left\|T^{*} x^{*}\right\|, \quad x^{*} \in X^{*},
$$

is an equivalent dual norm under which $\widetilde{X}=(X,|\cdot|)$ is still a canonical $(a, B, c)$ ideal in $\widetilde{X}^{* *}$

Moreover, if $T^{*}$ is injective and there is a strictly convex Banach space $Z$ such that $\operatorname{ran} T^{*} \subset Z \subset Y^{*}$, then $\widetilde{X}^{*}$ is strictly convex.

Proof. The proof is essentially the same as the proof of Theorem 3.1, except that here we need to show that $\widetilde{X}$ is a canonical $(a, B, c)$-ideal. This means that

$$
\left|a x^{* * *}+b \pi_{X} x^{* * *}\right|+c\left|\pi_{X} x^{* * *}\right| \leq\left|x^{* * *}\right| \quad \forall b \in B
$$

holds for all $x^{* * *}$ in $X^{* * *}$.

Let $x^{* * *} \in X^{* * *}$. Then $\left\|a x^{* * *}+b \pi_{X} x^{* * *}\right\|+c\left\|\pi_{X} x^{* * *}\right\| \leq\left\|x^{* * *}\right\|$ for all $b \in B$. Therefore, recalling that on $X^{* * *}$, the norm $|\cdot|$ is of the form $x^{* * *} \mapsto\left|x^{* * *}\right|=$ 


$$
\begin{aligned}
& \left\|x^{* * *}\right\|+\left\|T^{* * *} x^{* * *}\right\| \text { and } T^{* * *} \pi_{X}=T^{* * *} \text {, we have } \\
& \left|a x^{* * *}+b \pi_{X} x^{* * *}\right|+c\left|\pi_{X} x^{* * *}\right| \\
& =\left\|a x^{* * *}+b \pi_{X} x^{* * *}\right\|+\left\|a T^{* * *} x^{* * *}+b T^{* * *} \pi_{X} x^{* * *}\right\| \\
& +c\left\|\pi_{X} x^{* * *}\right\|+c\left\|T^{* * *} \pi_{X} x^{* * *}\right\| \\
& =\left\|a x^{* * *}+b \pi_{X} x^{* * *}\right\|+c\left\|\pi_{X} x^{* * *}\right\| \\
& +\left\|a T^{* * *} x^{* * *}+b T^{* * *} x^{* * *}\right\|+c\left\|T^{* * *} x^{* * *}\right\| \\
& \leq\left\|x^{* * *}\right\|+|a+b|\left\|T^{* * *} x^{* * *}\right\|+c\left\|T^{* * *} x^{* * *}\right\| \\
& \leq\left\|x^{* * *}\right\|+\left\|T^{* * *} x^{* * *}\right\|=\left|x^{* * *}\right| \text {, }
\end{aligned}
$$

because $|a+b|+c \leq 1$ for every $b \in B$ (this can easily be verified by considering an arbitrary $x^{*} \in S_{X^{*}}$.

Our next result extends [FG, renorming result on p. 142 after Theorem 3] and [HWW, Theorem III.4.6(e)] from $M$-ideals to strict $(a, B, c)$-ideals. Note that Theorem 4.3 applies in particular to $u$ - and $h$-ideals.

Theorem 4.3. Let $a, c \geq 0$, and let $B$ be a compact set of scalars. Assume that a Banach space $X$ is a strict $(a, B, c)$-ideal in $X^{* *}$. If $X$ has a shrinking Markushevich basis, then $X$ admits an equivalent smooth norm whose dual norm is strictly convex and under which $X$ becomes a canonical $(a, B, c)$-ideal in its bidual.

The proof of Theorem 4.3 uses the following result that relies on GK and extends GKS, Proposition 5.2, (1) and (2)], where $u$ - and $h$-ideals were considered.

Proposition 4.4. Let a Banach space $X$ be a strict $(a, B, c)$-ideal in $X^{* *}$. If $X$ does not contain $l_{1}$ isomorphically, then $X$ is a canonical $(a, B, c)$-ideal in $X^{* *}$.

PROOF. The proof follows the scheme of the proof of GKS Proposition 5.2, (1) and (2)].

By assumption, there is an $(a, B, c)$-ideal projection $P$ on $X^{* * *}$ such that ker $P=$ $X^{\perp}$ and $\operatorname{ran} P$ is a norming subspace of $X^{* * *}$. It suffices to show that $\operatorname{ran} P=X^{*}$, because then $P=\pi_{X}$ (recall that $\operatorname{ker} \pi_{X}=X^{\perp}$ and $\operatorname{ran} \pi_{X}=X^{*}$ ), meaning that $X$ is a canonical $(a, B, c)$-ideal in $X^{* *}$.

Since $X$ does not contain $l_{1}$ isomorphically, we get from GK, Corollary 5.5] that $X^{* * *}$ contains a minimal norming subspace, which is, by definition, the intersection of all norming subspaces of $X^{* * *}$. We know that $X^{*}$ is norming in $X^{* * *}$. On the other hand, a proper subspace $U$ of $X^{*}$ cannot be norming in $X^{* * *}$. Indeed, assume, for the sake of contradiction, that such a $U$ is norming in $X^{* * *}$. By the Hahn-Banach theorem, there is $x^{* *} \in X^{* *}$ such that $x^{* *}(u)=0$ for every $u \in U$, but $x^{* *} \neq 0$. Since $U$ is norming in $X^{* * *}$, we get

$$
\left\|x^{* *}\right\|=\sup _{u \in B_{U}}\left|x^{* *}(u)\right|=0,
$$

which is a contradiction.

Therefore, $X^{*}$ is the minimal norming subspace, and thus $X^{*} \subset \operatorname{ran} P$. Since now also $\operatorname{ran} P \subset X^{*}$ (indeed, if $x^{* * *}=x^{*}+x^{\perp} \in X^{* * *}$ with $x^{*} \in X^{*}, x^{\perp} \in X^{\perp}$, then $P x^{* * *}=P x^{*}=x^{*}$, because $X^{*} \subset \operatorname{ran} P$ ), we have $\operatorname{ran} P=X^{*}$, as desired.

Proof of Theorem 4.3. Since $X$ has a shrinking Markushevich basis, by Theorem 2.2 $X$ is Asplund. An Asplund space cannot contain $l_{1}$ isomorphically, and thus, by Proposition 4.4, $X$ is a canonical $(a, B, c)$-ideal. 
Now, using Theorem 4.2 (instead of Theorem 3.1) in the proof of Theorem 3.2 this immediately yields an equivalent smooth norm $|\cdot|$ on $X$ whose dual norm is strictly convex and for which $X$ is still a canonical $(a, B, c)$-ideal in its bidual.

If the latter proof is carried out under the supplementary assumption of property $U$, then one obtains the following partial positive answer to Problem 4.1, which is quite similar to Theorem 3.2

Theorem 4.5. Let $a, c \geq 0$, and let $B$ be a compact set of scalars. Assume that a Banach space $X$ is a strict $(a, B, c)$-ideal with property $U$ in $X^{* *}$. If $X$ is $W C G$, then $X$ has a shrinking Markushevich basis and $X$ admits an equivalent very smooth norm under which $X$ becomes a totally smooth canonical $(a, B, c)$-ideal in its bidual.

For separable spaces, we can again omit the WCG-assumption.

Corollary 4.6. Let $a, c \geq 0$, and let $B$ be a compact set of scalars. Assume that a separable Banach space $X$ is a strict $(a, B, c)$-ideal with property $U$ in $X^{* *}$. Then $X$ admits an equivalent very smooth norm under which $X$ is a totally smooth canonical $(a, B, c)$-ideal in its bidual.

As it was recalled in Section 3, every Asplund $U^{*}$-space has a shrinking Markushevich basis. Since this property is preserved under isomorphisms, Theorem 4.5 immediately implies the following.

Corollary 4.7. Let $a, c \geq 0$ and let $B$ be a compact set of scalars. Assume that a Banach space $X$ is a strict $(a, B, c)$-ideal with property $U$ in $X^{* *}$. If $X$ is isomorphic to a $U^{*}$-space, then $X$ admits an equivalent very smooth norm under which $X$ is a totally smooth canonical $(a, B, c)$-ideal in its bidual.

Similarly to Theorem 4.5. we can apply Theorem 4.3 to obtain the following result. However, here we need to use a couple of auxiliary results from the literature.

Theorem 4.8. Let a Banach space $X$ be a strict $(a, B, c)$-ideal in $X^{* *}$ with $\max \{|b|: b \in B\}+c>1$. If $X$ is isomorphic to a $U^{*}$-space, then $X$ admits an equivalent smooth norm whose dual norm is strictly convex and under which $X$ becomes a canonical $(a, B, c)$-ideal in its bidual.

Proof. A $U^{*}$-space does not contain $l_{1}$ isomorphically. This fact was observed in [CN1, Proposition 4.1] as a direct consequence of [GKS, Proposition 2.6]. Hence, $X$ does not contain $l_{1}$ isomorphically, and therefore, by Proposition 4.4 our $(a, B, c)$ ideal $X$ is canonical. But canonical $(a, B, c)$-ideals with $B$ and $c$ as above are Asplund spaces (see [06, proof of Theorem 4.1] or [OZ, Lemma 4.2]). Hence, $X$ has a shrinking Markushevich basis, and Theorem 4.3 applies.

Note that the above $(a, B, c)$-ideal assumption is satisfied in all important cases, including $M-, u^{-}$, and $h$-ideals.

Clearly, $M$-ideals in their biduals and, more generally, the canonical $(1,\{-1\}, c)$ ideals with $c \in(0,1]$ are $U^{*}$-spaces. Hence, from Theorem 4.8, we have the following example.

Example 4.9. Let $X$ be a canonical $(1,\{-1\}, c)$-ideal in $X^{* *}$ with $c \in(0,1]$. Then $X$ admits an equivalent smooth norm whose dual norm is strictly convex and under which $X$ is a canonical $(1,\{-1\}, c)$-ideal in its bidual. 
Remark 4.10. A particular example of a $(1,\{-1\}, c)$-ideal is provided by certain renormings of the James space $J$, as shown in [CN1, Example 3.5]. Namely, for $\delta>\sqrt{2}$ the renorming $J_{\delta}$ of the James space in [CN1, Example 3.5] is a canonical $(1,\{-1\}, c)$-ideal in $J_{\delta}^{* *}$ if

$$
\max \left\{\frac{(1+\delta c)^{2}}{\delta^{2}}, \frac{(1+\delta c)^{2}+(1+c)^{2}+2(\delta c)^{2}}{2 \delta^{2}}\right\}<\frac{1}{2} .
$$

(We take this opportunity to point out a disturbing typo in CN1 where the denominator in the first item of the maximum is 2 instead of $\delta^{2}$.) It is easy to see that for each $c<1 / \sqrt{3}$, there is some $\delta>\sqrt{2}$ satisfying the above inequality.

The point of this remark is that the James space $J$ cannot be renormed to be an $M$-ideal in its bidual, since it is non-reflexive and its bidual is separable, being isomorphic to $J$. However, a non-reflexive space that is an $M$-ideal in its bidual contains a copy of $c_{0}$ (see $\mathrm{HL}$ or, e.g, HWW, Corollary III.3.7(a)]; hence the renormed James space $J_{\delta}$ is a nontrivial instance of Example 4.9.

Let us conclude the paper with a couple of examples where Corollary 4.7 applies.

It is known (see CN1, Proposition 2.2]) that the space in Example 3.5 is a canonical $u$-ideal in its bidual. Using Corollary 4.7 (instead of Theorem 3.2) in the proof of Example 3.5 allows us to strengthen this - using the same norm as in Example 3.5 - as follows. Note that canonical $u$-ideals in their biduals could be considered as the closest important weakenings of $M$-ideals in their biduals.

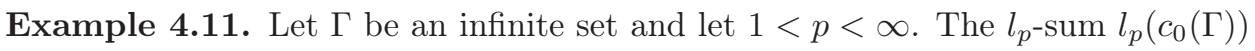
admits an equivalent very smooth norm under which it is a totally smooth canonical $u$-ideal in its bidual. But $l_{p}\left(c_{0}(\Gamma)\right)$ cannot be equivalently renormed to be an $M$ ideal in its bidual.

Our last Example 4.12 will concern a large class of spaces. We need some more notation. For Banach spaces $X$ and $Y$, we denote by $\mathcal{L}(X, Y)$ the Banach space of bounded linear operators from $X$ to $Y$, and by $\mathcal{K}(X, Y)$ its subspace of compact operators. We write $\mathcal{L}(X)$ and $\mathcal{K}(X)$, respectively, if $X=Y$. Recall that a net $\left(K_{\alpha}\right)$ in $\mathcal{K}(X)$ is a compact approximation of the identity $(\mathrm{CAI})$ provided $K_{\alpha} x \rightarrow x$ for all $x \in X$. If, moreover, $K_{\alpha}^{*} x^{*} \rightarrow x^{*}$ for all $x^{*} \in X^{*}$, then $\left(K_{\alpha}\right)$ is called a shrinking $C A I$. If $X$ has a CAI such that the convergence is uniform on compact subsets of $X$, then $X$ is said to have the compact approximation property (CAP).

Let $1<p<\infty$. A Banach space $X$ is said to have the upper p-property (cf. OW, Proposition 1.1] or [HWW, pp. 306 and 327]) if $X$ admits a shrinking CAI $\left(K_{\alpha}\right)$ such that

$$
\limsup \sup _{\alpha, y \in B_{X}}\left\|K_{\alpha} x+\left(y-K_{\alpha} y\right)\right\| \leq\left(\|x\|^{p}+\|y\|^{p}\right)^{1 / p} .
$$

If $X$ and $Y$ are both reflexive Banach spaces, and $X$ or $Y$ has the CAP, then $\mathcal{K}(X, Y)^{* *}=\mathcal{L}(X, Y)$ (see GS, Corollary 1.3]; in the AP-case, this is a well-known result due to Grothendieck). There is a vast literature studying the position of $\mathcal{K}(X, Y)$ in $\mathcal{L}(X, Y)$ in terms of ideals (see, e.g., [HJO for recent results and a large set of references). We are going to use [CN2, Theorem 4.4 and Corollary 4.5], from which one can see that $\mathcal{K}(X, Y)$ is a $U^{*}$-space with property $U$ and also a strict $(a,\{-a\}, c)$-ideal for all $a, c>0$ such that $a^{p}+c^{p} \leq 1$ in $\mathcal{L}(X, Y)$, whenever $X$ is an arbitrary Banach space and $Y$ is a Banach space having the upper $p$-property. Thanks to Corollary 4.7 we have the following rather general example. 
Example 4.12. Let $X$ and $Y$ be reflexive Banach spaces and let $1<p<\infty$. If $Y$ has the upper $p$-property, then $\mathcal{K}(X, Y)$ admits an equivalent very smooth norm under which $\mathcal{K}(X, Y)$ is a totally smooth canonical $(a,\{-a\}, c)$-ideal in its bidual for all $a, c>0$ such that $a^{p}+c^{p} \leq 1$.

Besides the $l_{p}(\Gamma)$ spaces and the Lorentz sequence spaces $d(w, p)$, there are many reflexive spaces enjoying the upper $p$-property, as one can see from [HWW, pp. 306, 327]. In the context of Example 4.12, we are interested in cases when $\mathcal{K}(X, Y)$ is not an $M$-ideal in its bidual $\mathcal{L}(X, Y)$. Here the classical example, due to Hennefeld (see [ $\underline{\mathrm{H}}$ or, e.g., [HWW, p. 305]), is that $\mathcal{K}(d(w, p))$ is not an $M$-ideal in its bidual $\mathcal{L}(d(w, p))$. If $1<p \leq q<\infty$, then $\mathcal{K}\left(l_{p}(\Gamma), d(w, q)\right)$ is an $M$-ideal in $\mathcal{L}\left(l_{p}(\Gamma), d(w, q)\right)$ by $\mathrm{O} 1$ (see, e.g., O4, p. 53 or p. 66]). However, in O3 (see, e.g., [4, p. 73 and p. 77]), it is proved that for $1<q<p<\infty$ and an infinite set $\Gamma$, $\mathcal{K}\left(l_{p}(\Gamma), d(w, q)\right)$ is not an $M$-ideal in $\mathcal{L}\left(l_{p}(\Gamma), d(w, q)\right)$ whenever $w \in l_{\frac{p}{p-q}}$, neither is $\mathcal{K}\left(d(v, p)^{*}, d(w, q)\right)$ in $\mathcal{L}\left(d(v, p)^{*}, d(w, q)\right), 1<p, q<\infty$, whenever $p>(p-1) q$ and $d(v, p)^{*}$ (which is a sequence space) is contained as a linear subspace in $d(w, q)$.

Acknowledgements. This research was partially supported by institutional research funding IUT20-57 of the Estonian Ministry of Education and Research and by the German Academic Exchange Service (DAAD).

\section{REFERENCES}

[AE] E. M. Alfsen and E.G. Effros, Structure in real Banach spaces. Parts I and II, Ann. Math. 96 (1972), 98-173.

[AL] D. Amir and J. Lindenstrauss, The structure of weakly compact sets in Banach spaces, Ann. Math. 88 (1968), 35-46.

[CN1] J. C. Cabello and E. Nieto, On properties of M-ideals, Rocky Mount. J. Math. 28 (1998), 61-93.

[CN2] J. C. CABello And E. Nieto, An ideal characterization of when a subspace of certain Banach spaces has the metric compact approximation property, Studia Math. 129 (1998), 185-196.

[Da] M. M. DAY, Strict convexity and smoothness of normed spaces, Trans. Amer. Math. Soc. 78 (1955), 516-528.

[Di] J. Diestel, Geometry of Banach Spaces - Selected Topics, Lecture Notes in Math. 485, Springer, Berlin-Heidelberg-New York, 1975.

[DF] J. Diestel and B. Faires, On vector measures, Trans. Amer. Math. Soc. 198 (1974), 253-271.

[Fa] M. Fabian, Gâteaux Differentiability of Convex Functions and Topology: Weak Asplund Spaces, Canadian Mathematical Society Series of Monographs and Advanced Texts, Wiley, New York, 1997.

[FG] M. Fabian and G. Godefroy, The dual of every Asplund space admits a projectional resolution of identity, Studia Math. 91 (1988), 141-151.

[Fo] S. R. Foguel, On a theorem by A. E. Taylor, Proc. Amer. Math. Soc. 9 (1958), 325.

[G] G. Godefroy, Points de Namioka, espaces normants, applications à la théorie isométrique de la dualité, Israel J. Math. 38 (1981), 209-220.

[GK] G. Godefroy and N. J. Kalton, The ball topology and its applications, Proceedings of the Iowa Workshop on Banach spaces, Contemp. Math. 85 (1989), 195-238.

[GKS] G. Godefroy, N. J. Kalton, and P. D. Saphar, Unconditional ideals in Banach spaces, Studia Math. 104 (1993), 13-59.

[GS] G. Godefroy And P. D. Saphar, Duality in spaces of operators and smooth norms on Banach spaces, Illinois J. Math. 32 (1988), 672-695.

[H] J. Hennefeld, M-ideals, HB-subspaces, and compact operators, Indiana Univ. Math. J. 28 (1979), 927-934.

[HJO] R. Haller, M. Johanson, and E. OJa, $M(r, s)$-ideals of compact operators, Czechoslovak Math. J. 62 (2012), 673-693. 
[HL] P. Harmand and A. Lima, Banach spaces which are $M$-ideals in their biduals, Trans. Amer. Math. Soc. 283 (1984), 253-264.

[HR] P. HaRmand And T. S. S. R. K. RaO, An intersection property of balls and relations with M-ideals, Math. Z. 197 (1988), 277-290.

[HWW] P. Harmand, D. Werner, And W. Werner, M-ideals in Banach Spaces and Banach Algebras, Lecture Notes in Math. 1547, Springer, Berlin, 1993.

[Ka] M. I. Kadets, On weak and norm convergence, Dokl. Akad. Nauk. SSSR 122 (1958), 13-16 (in Russian).

[K11] V. L. KLeE, Convex bodies and periodic homeomorphisms in Hilbert space, Trans. Amer. Math. Soc. 74 (1953), 10-43.

[Kl2] V. L. KleE, Mappings into normed linear spaces, Fund. Math. 49 (1960), 25-34.

[LT1] J. Lindenstrauss and L. Tzafriri, Classical Banach Spaces I, Springer, BerlinHeidelberg-New York, 1977.

[LW] C.-J. Liao and N.-C. Wong, Smoothly embedded subspaces of a Banach space, Taiwanese J. Math. 14 (2010), 1629-1634

[O1] E. OJA, On the uniqueness of the norm-preserving extension of a linear functional in the Hahn-Banach theorem, Izv. Akad. Nauk. Est. SSR 33 (1984), 424-438 (in Russian).

[O2] E. OJA, Strong uniqueness of the extension of linear continuous functionals according to the Hahn-Banach theorem, Math. Notes 43 (1988), 134-139.

[O3] E. OJA, On M-ideals of compact operators and Lorentz sequence spaces, Proc. Est. Acad. Sci. Phys. Math. 40 (1991), 31-36.

[O4] E. OJA, Extensions of Functionals and the Structure of the Space of Continuous Linear Operators, Tartu Univ. Publ., Tartu, 1991 (in Russian).

[O5] E. OJA, Géométrie des espaces de Banach ayant des approximations de l'identité contractantes, C. R. Acad. Sci. Paris Sér. I Math. 328 (1999), 1167-1170.

[O6] E. OJA, Geometry of Banach spaces having shrinking approximations of the identity, Trans. Amer. Math. Soc. 352 (2000), 2801-2823.

[OP] E. OJa And M. PÕldvere Norm-preserving extensions of functionals and denting points of convex sets, Math. Z. 258 (2008), 333-345.

[OPV] E. Oja, M. Põldvere, ANd T. Vill, On totally smooth subspaces of Banach spaces: the Vlasov theorem revisited, Studia Math. 238 (2017), 91-99.

[OW] E. OJA And D. Werner, Remarks on $M$-ideals of compact operators on $X \oplus_{p} X$, Math. Nachr. 152 (1991), 101-111.

[OZ] E. OJA AND I. ZoLK, On commuting approximation properties of Banach spaces, Proc. Royal Soc. Edinb. 139A (2009), 551-565.

[P] R. R. Phelps, Uniqueness of Hahn-Banach extensions and unique best approximation, Trans. Amer. Math. Soc. 95 (1960), 238-255.

[SS] M. A. Smith And F. Sullivan, Extremely smooth Banach spaces, in: Banach Spaces of Analytic Functions, Proc. Conf. Kent, Ohio, 1976, J. Baker, C. Cleaver and J. Diestel (eds.) Lecture Notes in Math. 604, Springer, Berlin 1977, 125-137.

[S] F. Sullivan, Geometrical properties determined by the higher duals of a Banach space, Illinois J. Math. 21 (1977), 315-331.

[T] A. E. TAYLOR, The extension of linear functionals, Duke Math. J. 5 (1939), 538-547.

(Oja) Institute of Mathematics and Statistics, University of Tartu, J. Lini 2, 50409 Tartu, Estonia, and Estonian Academy of Sciences, Kohtu 6, 10130 Tallinn, ESTONIA

E-mail address: eve.॰ja@ut.ee

(Viil) Institute of Mathematics and Statistics, University of Tartu, J. Livi 2, 50409 Tartu, Estonia

E-mail address: tauriv@ut.ee

(Werner) Department of Mathematics, Freie Universität Berlin, Arnimallee 6, 14195 Berlin, Germany ORCID: 0000-0003-0386-9652

E-mail address: werner@math.fu-berlin.de 